\title{
Determining the molecular landscape and impact on prognosis in HPV-associated head and neck cancer
}

\author{
Suchin Khanna ${ }^{\text {** }}$, Sarah Palackdharry², Logan Roof ${ }^{2}$, Christina A. Wicker ${ }^{3}$, Jonathan Mark ${ }^{4}$, Zheng Zhu ${ }^{5}$, \\ Roman Jandorav ${ }^{5}$, Alfredo Molinolo ${ }^{6}$, Vinita Takiar ${ }^{3}$ and Trisha M. Wise-Draper ${ }^{2}$
}

\begin{abstract}
Background: Human papillomavirus (HPV) associated head and neck squamous cell carcinoma (HNSCC) has a better prognosis than HNSCC due to other risk factors. However, there is significant heterogeneity within HPV-associated HNSCC and 25\% of these patients still do poorly despite receiving aggressive therapy. We currently have no good molecular tools to differentiate and exclude this "high-risk" sub-population and focus on "low-risk" patients for clinical trials. This has been a potential barrier to identifying successful de-escalation treatment strategies in HPV-associated HNSCC. We conducted an analysis of molecular markers with a well-known role in the pathogenesis of HPV-associated HNSCC and hypothesized that these markers could help independently predict recurrence and prognosis in these patients and therefore help identify at the molecular level "low-risk" patients suitable for de-escalation trials.

Methods: We analyzed 24 tumor specimens of patients with p16+ HNSCC who underwent definitive resection as primary treatment. Tissue microarray (TMA) was generated from the 24 pathology blocks and immunohistochemistry (IHC) was performed using highly specific antibodies for our chosen biomarkers (PI3K-PTEN, AKT pathway, mTOR, 4EBP1, S6, and PAMPK, ERCC-1). Transcriptome data was also obtained for 7 p16+ HNSCC patients from The Cancer Genome Atlas (TCGA). Data from the TMA and TCGA were analyzed for association of relapse-free survival (RFS) and overall survival (OS) with protein and gene expression of the chosen biomarkers.
\end{abstract}

Results: Increased pAMPK protein activity by IHC and AMPK gene expression by TCGA gene expression data was correlated with improved RFS with a trend towards statistical significance.

Conclusions: This data suggests that increased PAMPK activity and expression may portend a better prognosis in HPVassociated HNSCC undergoing primary definitive resection. However, these findings require validation in larger studies.

Keywords: Head and neck, Oropharynx, Molecular biology, Human papillomavirus, pAMPK

\section{Background}

Head and neck squamous cell carcinoma (HNSCC) is the sixth most common cancer type worldwide, accounting for more than 550,000 cases and 380,000 deaths annually [1]. Human papillomavirus (HPV) is a significant risk factor and is particularly prevalent in oropharyngeal squamous

\footnotetext{
* Correspondence: Suchin.Khanna@yale.edu

'Division of Hematology/Oncology, Department of Internal Medicine, Yale University School of Medicine, 333 Cedar Street WWW 201 Attn: Suchin

Khanna, New Haven, CT 06510, USA

Full list of author information is available at the end of the article
}

cell carcinomas (OPSCC). The incidence of OPSCC has been on the rise over the past three decades, with multiple lines of evidence linking the rise of OPSCC incidence to the rise in HPV $[2,3]$. HPV-associated OPSCC is thought to be a distinct clinical and molecular entity with unique histopathological features as compared to tobacco and alcohol related HNSCC [4]. HPV-associated HNSCC has a better prognosis with improved survival and enhanced response to treatment, irrespective of treatment modality, compared to HNSCC due to other risk factors [5-7].

(c) The Author(s). 2020 Open Access This article is licensed under a Creative Commons Attribution 4.0 International License, which permits use, sharing, adaptation, distribution and reproduction in any medium or format, as long as you give appropriate credit to the original author(s) and the source, provide a link to the Creative Commons licence, and indicate if changes were made. The images or other third party material in this article are included in the article's Creative Commons licence, unless indicated otherwise in a credit line to the material. If material is not included in the article's Creative Commons licence and your intended use is not permitted by statutory regulation or exceeds the permitted use, you will need to obtain permission directly from the copyright holder. To view a copy of this licence, visit http://creativecommons.org/licenses/by/4.0/. 
Despite the different epidemiology, natural history and treatment response, HPV-associated OPSCC is currently managed in the same way as HPV-negative cancer, often with toxic multimodality therapy [8]. Patients can experience significant morbidity and diminished quality of life.

Numerous recent clinical trials have studied deescalation treatment strategies in HPV-associated OPSCC in the hope of reducing toxicities while maintaining high cure rates, however none of these trials have been practice changing. In a multicenter cooperative group study, ECOG 1308, 80 patients with Stage III or IVA (using AJCC 7th Edition Staging Manual) HPV-associated OPSCC received induction chemotherapy with three cycles of cisplatin, paclitaxel, and cetuximab followed by cetuximab combined with a lower dose of radiation therapy (RT), 54 Gy in 27 fractions, for those patients who achieved a clinical complete response to induction chemotherapy at the primary site. With the lower dose of radiation, the two-year progression-free survival (PFS) was $80 \%$ and two-year overall survival (OS) was 94\% [9]. These results are similar to those of the ECOG 2399 study, the Lancet Oncology study by Chen et al., and the OPTIMA study [10-12]. However, much like ECOG 1308 all of these studies included a small number of patients and therefore no definitive conclusion about this de-escalation strategy can be made without further data from larger studies.

A more recent and large randomized study, RTOG 1016 which studied concurrent chemoradiation with cetuximab versus cisplatin in HPV-associated OPSCC reported worse overall survival and progression-free survival for the hypothesized less toxic concurrent cetuximab/RT arm compared to cisplatin/RT and surprisingly and importantly also showed similar toxicity [13]. A similar trial comparing cetuximab/RT versus cisplatin/RT, TROG 12.01, is ongoing as are several other de-escalation trials [14-16] and it remains to be seen whether any of these trials will be practice changing. The DE-ESCALATE HPV trial, like RTOG 1016, also compared effectiveness and toxicity of cisplatin/RT versus cetuximab/RT in HPV-associated OPSCC. It showed consistent findings with RTOG 1016, namely that there was no benefit in terms of reduced toxicity with cetuximab but there was inferior tumor control and survival outcomes with cetuximab [17].

A potential barrier that RTOG 1016, DE-ESCALATE $\mathrm{HPV}$, and other similar trials are experiencing is that HPV-associated OPSCC is a heterogeneous disease with up to $25 \%$ of these patients still doing poorly despite receiving aggressive therapy. These $25 \%$ of patients are therefore unsuitable candidates for de-escalation strategies, however we currently have no way of identifying this sub-population to exclude them from trials. It is therefore possible that RTOG 1016 and other de-escalation studies have failed to maintain equivalent outcomes because these trials do not optimally risk-stratify HPV-positive patients. With improved means of identifying characteristics of the subset of HPV-associated OPSCC patients with better prognoses we might be able to better identify suitable candidates for de-escalation strategies in future trials.

The molecular basis of the difference in outcomes amongst the HPV-associated OPSCC subgroups remain elusive. Finding such molecular means of prognosticating and risk-stratifying HPV-associated OPSCC patients will be profoundly useful in the future to more accurately select suitable candidates for de-escalation treatment strategies as well as to determine appropriate primary modality of treatment. We conducted an analysis of molecular markers with these goals in mind. We specifically analyzed HPV-associated OPSCC patients that underwent definitive resection as their primary modality of treatment. We studied biomarkers with a well-known role in the molecular pathogenesis of HPV-associated OPSCC with the hypothesis that these markers could help predict recurrence and prognosis in these patients after surgery. These markers included proteins in the PI3K-PTEN-AKT pathway, mTOR, 4EBP1, S6, and pAMPK, ERCC-1 [18-22].

\section{Methods \\ Study participants}

This is an IRB-approved (University of Cincinnati IRB\#: 2016-0532) retrospective study that analyzed the tumor specimens of patients with p16+ OPSCC by immunohistochemistry who underwent definitive resection as primary treatment from May 2010 to April 2016. Inclusion criteria included adult patients with HPV-associated OPSCC that underwent definitive surgical resection and are followed at the University of Cincinnati. Exclusion criteria included patients with incomplete records, patients with prior history of HNSCC, patients with multiple simultaneous cancers, pregnant women, children (age $<18$ ), and prisoners. The following characteristics were obtained and accounted for in each patient: Age, Gender, Location of Tumor, TNM Staging of Tumor at time of definitive resection, Date of Definitive Resection, Date of Last Follow-Up, Recurrence Status, Date of Recurrence (if applicable), Date of Death (if applicable), Adjuvant Treatment (if any), Surgical Margin status, Lymphovascular Invasion (LVI), Perineural Invasion (PNI), Extracapsular Extension (ECE), Overall Survival (OS), and Relapse-Free Survival (RFS). Twenty-six resected tumor specimens from patients that met the criteria highlighted above were screened, but only 24 patients had sufficiently high-quality tissue staining for analysis. 


\section{Molecular markers}

We studied biomarkers with a well-known role in the molecular pathogenesis of HPV-associated OPSCC One of the most frequently altered pathways in cancer with mutations in up to $30 \%$ of HNSCC is the PI3K pathway which regulates physiologic cellular processes including cell proliferation, differentiation, motility, metabolism, and apoptosis. Its central components, PI3K, AKT, and mTOR, drive tumor metastasis by promoting cell motility. PTEN acts as a negative regulator of this pathway. Downstream effectors of the PI3K pathway include pS6 and 4EBP1 $[19,23]$. Head and neck tumors often increase glycolysis to generate ATP, even in the presence of normal oxygen concentrations since this is much faster than oxidative phorphorylation in order to meet the tumor's increased energy and biosynthesis needs. Aerobic glycolysis, as this process is called, is regulated by aberrant signaling pathways including the AMPK pathway [24]. ERCC-1 has been theorized to have a role in prognosis and response to treatment in HNSCC, and this biomarker was therefore also included in this study [22].

\section{Tissue microarray, immunohistochemistry}

A tissue microarray (TMA) was generated from the 24 pathology blocks with $1 \mathrm{~mm}$ core size. Tumor specimens were de-identified and sent for molecular analysis using immunohistochemistry (IHC). The collected tissues were dehydrated and embedded in paraffin following standard procedures. Hematoxylin-eosin stained $5 \mu \mathrm{m}$ sections were used. Unstained sections were deparaffinized and processed for IHC. IHC was performed using highly specific antibodies for our chosen biomarkers (Table 1). The antibodies used and the procedures carried out involved overnight incubation with the primary antibody, biotinylated secondary antibodies, and the $\mathrm{ABC}$ method to detect the presence of reactive antibodies recognizing each protein. Our pathologist who was blinded to any

Table 1 List of antibodies for immunohistochemistry of tumor samples

Antibody Targets

Phospho-AKT (Ser473)

Phospho-4E-BP1 (Thr37/46) (236B4)

Phospho-S6 Ribosomal Protein (Ser235/236)

ERCC1 (D6G6)

mTOR (7C10)

PTEN (138G6)

Phospho-mTOR (Ser2448) (49F9)

Phospho-AMPKa (Thr172) (40H9)

PI3 Kinase p110a (C73F8)

Cyclin D1 (92G2)

Phospho-AKT (Thr308) (244F9) clinical information independently analyzed the IHCstained biopsy slides. For every case, the whole tumor areas were quantified. The number of positive cells were visually evaluated for each tissue and the results were reexpressed as a percentage of stained cells out of the total number of cells. The intensity of immunoreactivity was also evaluated with scores of 1 for weak, 2 for moderate, and 3 for strong. An $\mathrm{H}$-score was then calculated by multiplying both parameters [25].

\section{The Cancer Genome Atlas}

Transcriptome data (mRNASeq2, level 3) was obtained from The Cancer Genome Atlas (TCGA). Data were analyzed for RFS with regards to gene expression of AMPK (PRKAA1), PI3K (PIK3CA), AKT1, AKT2, AKT3, ERCC1, S6 (RPS6), PTEN, mTOR, EIF4EP1 (4E-BP1).

\section{Statistical analysis for protein activity and gene expression analysis}

For protein expression analysis, data were analyzed using the Cox Proportional Hazards model for the primary outcomes, relapse-free survival (RFS) and overall survival (OS). RFS was defined as the duration of time from surgery to time of relapse and OS was defined as the duration of time from diagnosis until time of death. All hazard ratios and/or odds ratios and 95\% confidence intervals were reported. Kaplan-Meier curves were created, and comparisons of Kaplan-Meier survival data between different subgroups and the corresponding hazard functions were conducted by two-sided log-rank tests. $P$-values less than 0.05 were reported as indicators of significant difference between the subgroups. All analyses were conducted using R 3.1.3.

For gene expression analysis, Kaplan-Meier curves were created, and RFS was compared between individuals with mRNA levels $\geq$ the median and $<$ the median values for each given target. Log-rank $P$-values of less than 0.05 were reported as indicators of significant difference between the subgroups. Statistical analyses were conducted using R.3.6.3 "Holding the Windsock."

\section{Results}

\section{Patient characteristics}

For immunohistochemistry analysis, tumor tissue specimens from 26 patients that met eligibility criteria for this retrospective study were identified through the head and neck cancer tissue database in University of Cincinnati's Department of Pathology. Patient characteristics are summarized in Table 2A. The median age was 61.5 , with a range from 39 to 83 years of age. The majority of patients were male (89\%). About half of the patients studied were found to be Stage I at diagnosis (46\%) by the AJCC Eighth Edition Staging Manual, half of the patients were found to be Stage II (50\%), and 1 of the 26 
(4\%) patients was Stage III. Regarding high risk features, 8 of the 26 patients (31\%) were found to have positive surgical margins and 6 of the 26 patients (23\%) were found to have extracapsular extension (ECE) after surgical resection. A total of 12 patients (46\%) had at least one high-risk feature (either ECE or positive surgical margins). Many of the patients were also noted to have intermediate risk features such as lymphovascular invasion (31\%), perineural invasion (12\%), T3/T4 disease (15\%), or N2/N3 disease (46\%). Overall, 5 patients $(19 \%)$ had intermediate risk disease. Median follow-up was 18.3 months. Fourteen percent of the patients had recurred and $11 \%$ had died at the time the retrospective study was conducted. Approximately $40 \%$ of patients received adjuvant treatment after definitive surgery, specifically those with high-risk (adjuvant concurrent chemoradiation) and intermediate-risk (adjuvant radiation therapy only) disease.

For gene expression analysis, transcriptome data was obtained from The Cancer Genome Atlas (TCGA). Patient characteristics are summarized in Table 2B. There were $7 \mathrm{p} 16+$ patients with recurrence data. The median age was 58, with a range from 47 to 68 years of age. All of the patients were male. Staging data was determined by the AJCC Seventh Edition Staging Manual. One (14.3\%) patient was Stage I, $6(85.7 \%)$ were stage IVA/B. Two (14.3\%) were T1, 1 (14.3\%) was T2 and 4 (57.1\%) were T4a. One patient (14.3\%) was N0, 4 (57.1\%) were N2/2b, 1 (14.3\%) was N3. Three (42.9\%) of patients had confirmed recurrence. All patients were alive at last follow-up. Five (71.4\%) tumors originated from the base of tongue (BOT) and $2(28.6 \%)$ originated from the tonsils.

\section{Survival stratified by tumor risk features}

Kaplan-Meier curves for RFS and OS for the entire cohort of 26 patients are included in Fig. 1a and b, respectively. As expected, prognosis of these patients was good with over $90 \%$ OS at 3 years. Three-year RFS was $73 \%$. RFS for patients with high-risk features was $67 \%$ while those with low- or intermediate-risk features had a 3year RFS of $79 \%$ (Fig. 2a). Importantly, this translated into a 100\% 3-year OS for low or intermediate-risk patients compared to $83 \%$ in those with high risk features (Fig. 2b). Although these findings were not statistically significant likely due to small sample size, it does suggest that pathological high-risk features in HPV-positive disease are prognostically similar to those with HPVnegative disease. Understanding those at high risk for relapse after surgical resection therefore is important for risk stratification and consideration of alternative treatment modalities.
Table 2 Characteristics of patients in the study sample. A) Patient characteristics for immunohistochemistry analysis. B) Patient characteristics for TCGA gene expression analysis

\begin{tabular}{|l|l|c|}
\hline \multicolumn{2}{|l|}{$\begin{array}{c}\text { A. Patient Characteristics for } \\
\text { Immunohistochemistry Analysis }\end{array}$} & $\begin{array}{c}\text { Study Cohort } \\
\text { (n = 26) }\end{array}$ \\
\hline Age (median, years) & $\begin{array}{c}61.5 \\
\text { (range 39-83) }\end{array}$ \\
\hline Male & $23(88.5 \%)$ \\
\hline Female & I & $3(11.5 \%)$ \\
\hline \multirow{5}{*}{ Stage } & II & $12(46.2 \%)$ \\
\cline { 2 - 3 } & III & $13(50 \%)$ \\
\cline { 2 - 3 } & T3/T4 & $1(3.8 \%)$ \\
\cline { 2 - 3 } & N2/N3 & $4(15.4 \%)$ \\
\hline \multirow{4}{*}{ Resection Status } & Positive Margins & $8(30.8 \%)$ \\
\cline { 2 - 3 } & ECE & $6(23.1 \%)$ \\
\cline { 2 - 3 } & LVI & $8(30.8 \%)$ \\
\cline { 2 - 3 } & PNI & $3(11.5 \%)$ \\
\hline Recurrence Confirmed & $5(19.2 \%)$ \\
\hline Number of Deaths & $3(11.5 \%)$ \\
\hline Adjuvant Treatment Received & $10(38.5 \%)$ \\
\hline
\end{tabular}

\begin{tabular}{|c|c|c|}
\hline \multicolumn{2}{|c|}{$\begin{array}{l}\text { B. Patient Characteristics for } \\
\text { TCGA Gene Expression Analysis }\end{array}$} & $\begin{array}{l}\text { Study Cohort } \\
\qquad(n=7)\end{array}$ \\
\hline \multicolumn{2}{|c|}{ Age (median, years) } & $\begin{array}{c}58 \\
\text { (range } 47-68 \text { ) }\end{array}$ \\
\hline \multicolumn{2}{|l|}{ Male } & $7(100 \%)$ \\
\hline \multicolumn{2}{|l|}{ Female } & $0(0 \%)$ \\
\hline \multirow{8}{*}{ Stage } & II & $1(14.3 \%)$ \\
\hline & IVAVB & $6(85.7 \%)$ \\
\hline & $\mathrm{T} 1$ & $2(28.6 \%)$ \\
\hline & T2 & $1(14.3 \%)$ \\
\hline & $\mathrm{T} 4 \mathrm{a}$ & $4(57.1 \%)$ \\
\hline & NO & $1(14.3 \%)$ \\
\hline & $\mathrm{N} 2 / 2 \mathrm{~b}$ & $4(57.1 \%)$ \\
\hline & N3 & $1(14.3 \%)$ \\
\hline \multicolumn{2}{|c|}{ Recurrence Confirmed } & $3(42.9 \%)$ \\
\hline \multicolumn{2}{|c|}{ Number of Deaths } & $0(0 \%)$ \\
\hline \multirow{2}{*}{ Tumor Site } & Base of Tongue & $5(71.4 \%)$ \\
\hline & Tonsil & $2(28.6 \%)$ \\
\hline
\end{tabular}




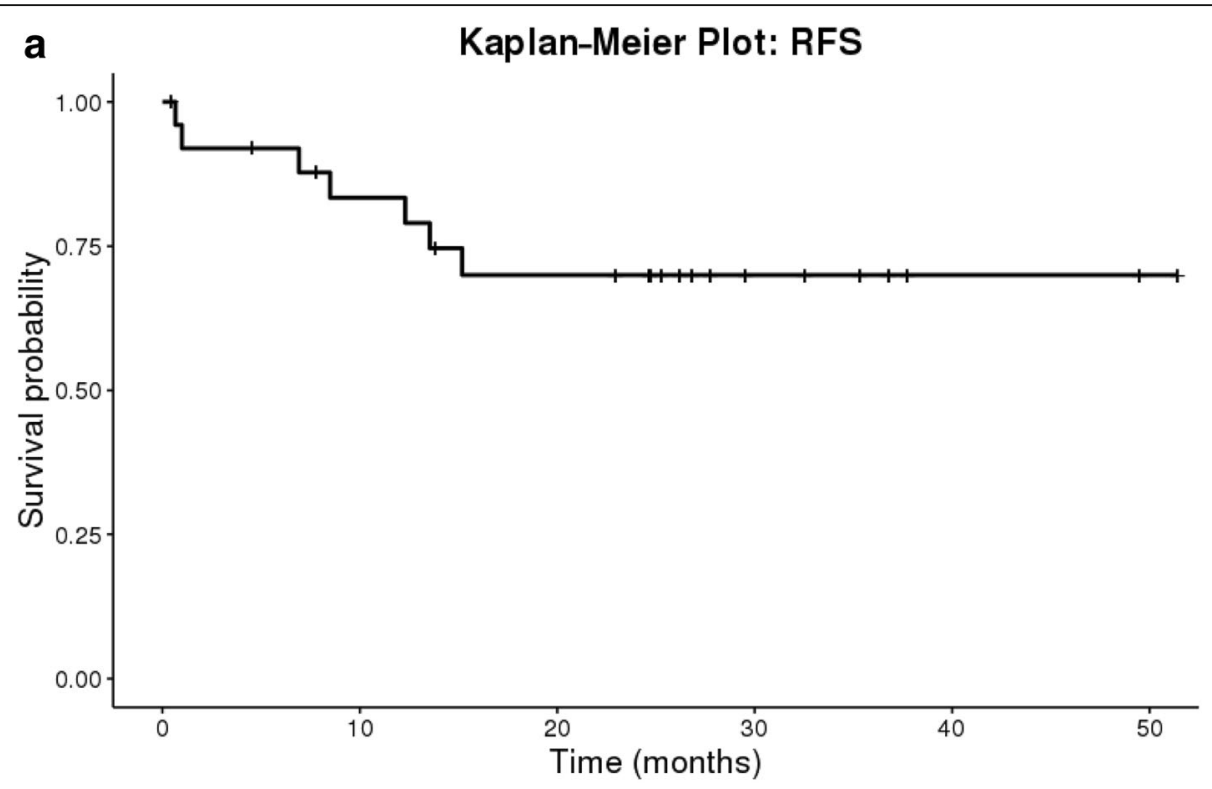

Number at risk

All \begin{tabular}{cccccc}
26 & 19 & 15 & 7 & 2 & 1 \\
\hline 0 & 10 & 20 & Time (months) & 10 & 50
\end{tabular}

b

Kaplan-Meier Plot: OS

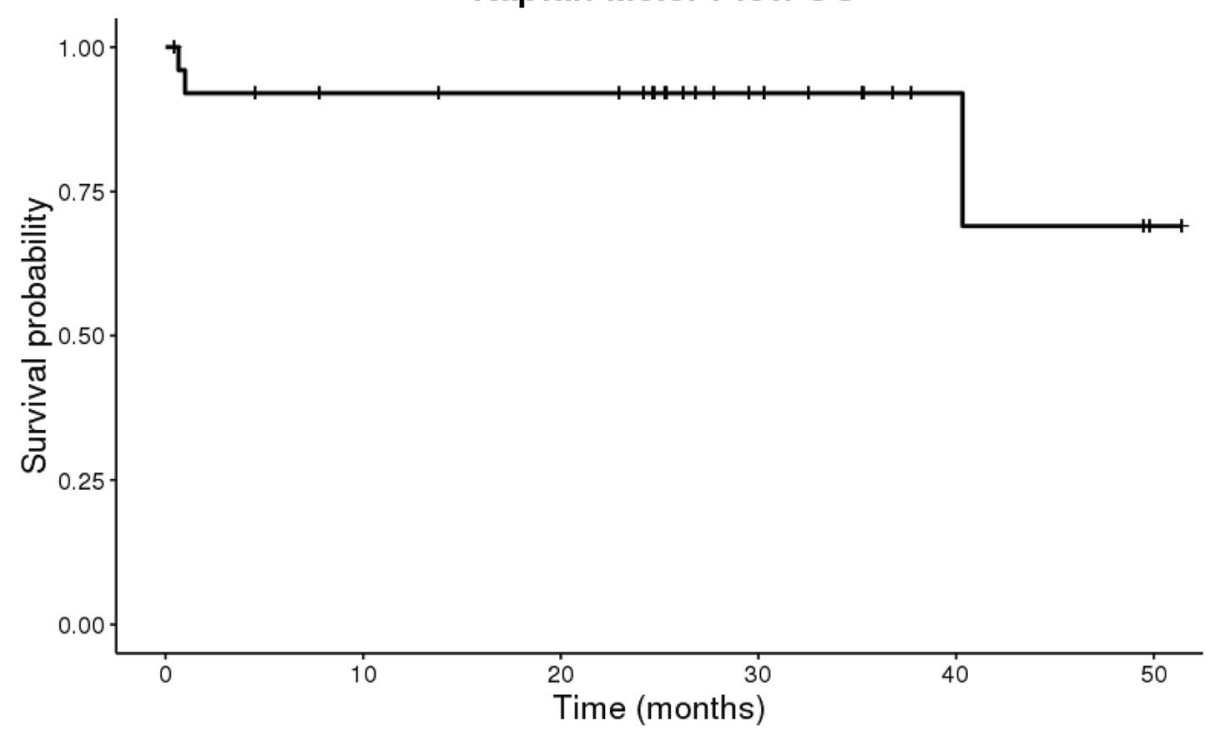

Number at risk

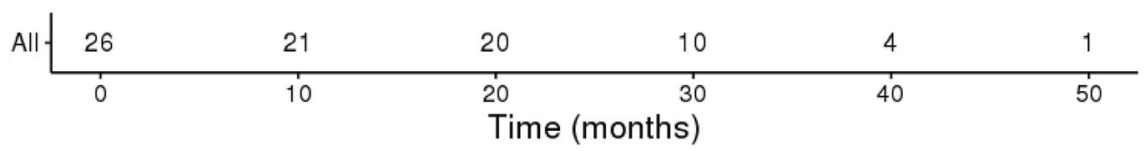

Fig. 1 a Kaplan Meier Curve for relapse-free survival for the total study sample. b Kaplan Meier Curve for overall survival for the total study sample 


\section{a \\ Kaplan-Meier Plot by risk: RFS}

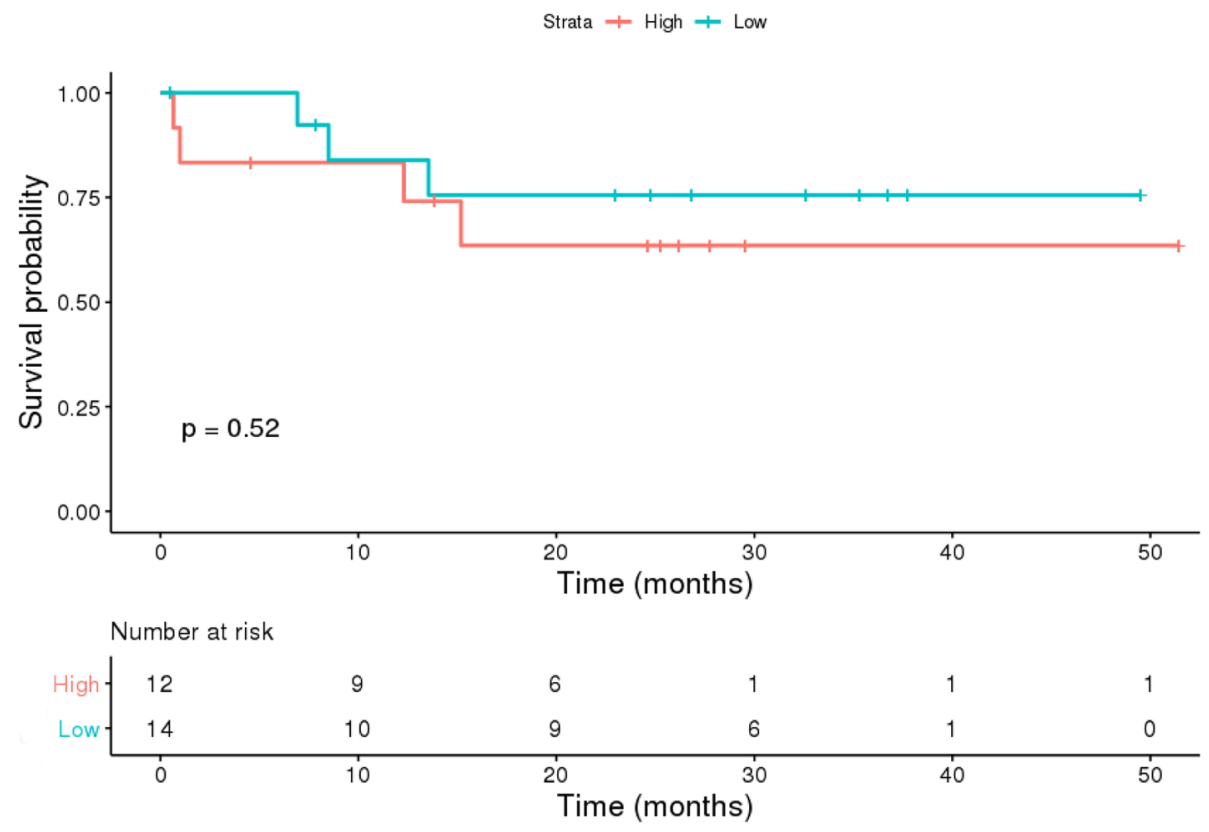

b

Kaplan-Meier Plot by risk: OS
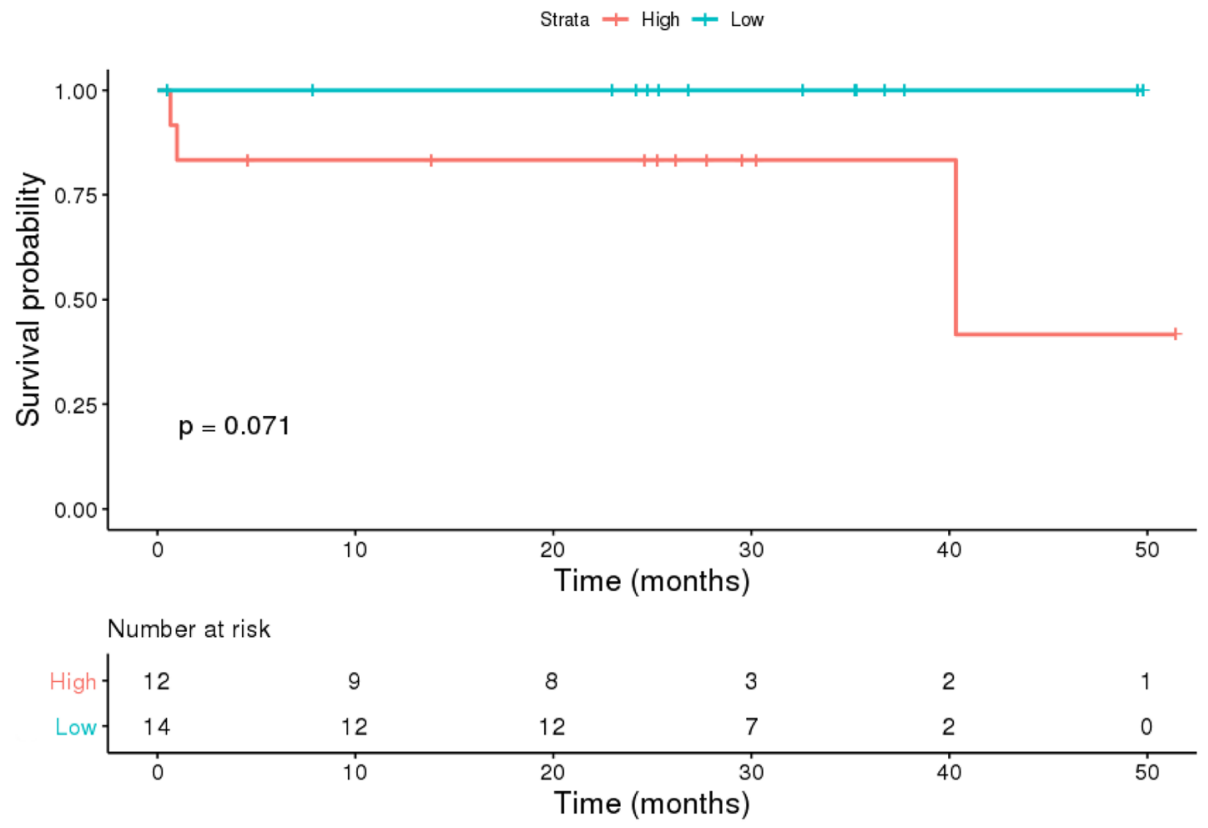

Fig. 2 a Kaplan-Meier Curves for relapse-free survival for high-risk (red) versus lowrisk (blue) patients in our study sample. b Kaplan Meier Curves for overall survival for high-risk (red) versus lowrisk (blue) patients in our study sample

\section{Recurrence and survival by biomarker analysis}

Molecular biomarker analysis was assessed by immunohistochemistry. Although 26 patients were selected for tissue microarray construction, only 24 patients had quality tissue staining for analysis. Protein expression was scored based on staining intensity and percentage cells that stained positive in each pathology specimen and was correlated with RFS and OS. Figure 3 illustrates representative pAMPK immunostaining in the TMA representing sample patients with low, intermediate and high expression. Interestingly, although not statistically significant, increased PAMPK expression trended 
towards statistical significance with improved RFS with a hazard ratio of 0.004 and $p=0.093$ (Table 3). pAMPK expression was not correlated with OS with a hazard ratio of $1.1(p=0.99)$. The other molecular markers, including ERCC1, 4EBP1, mTOR, PI3K, PTEN, AKT, and S6 all had hazard ratios very close to 1 for both RFS and OS without statistical significance. There was no discernible correlation between pAMPK expression and surgical margin status, ECE, PNI, or LVI (Table 4). There was also no correlation between expression of any of the other molecular markers and surgical margin status, ECE, PNI, or LVI.

Molecular biomarker analysis was also assessed using TCGA gene expression data. Only 7 P16 positive patients had recurrence data recorded. RFS was assessed between individuals with increased ( $\geq$ median) and decreased $(<$ median) gene expression (Supplemental Figure 1). Though not statistically significant, patients with increased AMPK gene expression had greater RFS as compared to patients with lower AMPK gene expression $(p=0.19)$ (Supplemental Figure 1A). This was similar to the correlation between superior RFS and increased protein expression of AMPK in our cohort. Patients with increased PI3K gene expression had increased RFS $(p=0.13)$ and patients with increased AKT1 gene expression trended toward reduced RFS ( $p=0.073$ ) (Supplemental Figure 1B,C). RFS curves were also plotted for ERCC1, S6 (RPS6), PTEN, AKT2, AKT3, mTOR, EIF4EP1 (4E-BP1).

\section{Discussion}

Patients with HPV-associated OPSCC often experience significant morbidity with treatment, and in most
Table 3 Correlation between biomarker protein activity and RFS and OS

\begin{tabular}{lllll}
\hline Risk Factor & RFS HR & $P$-Value & OS HR & $P$-Value \\
\hline pAMPK & 0.004 & 0.093 & 1.14 & 0.999 \\
ERCC1 & 1.001 & 0.954 & 1.043 & 0.361 \\
p4EBP1 & 1.000 & 0.982 & 1.010 & 0.508 \\
pmTOR & 1.023 & 0.181 & 1.042 & 0.209 \\
PI3K & 0.980 & 0.091 & 0.990 & 0.401 \\
PTEN & 0.993 & 0.116 & 1.004 & 0.744 \\
pAkt & 0.982 & 0.103 & 0.992 & 0.568 \\
pS6 & 1.002 & 0.769 & 0.761 & 0.998 \\
\hline
\end{tabular}

circumstances treatment includes multiple different modalities. Primary treatment often involves surgery or radiation therapy for localized early stage disease, and surgery followed by adjuvant therapy (radiation therapy if intermediate risk features such as LVI, PNI, or T3/T4 or N2/ N3 disease; concurrent chemoradiation for high risk features such as positive surgical margins or ECE) versus primary concurrent chemoradiation for locally advanced disease. Although testing for HPV positivity provides prognostic information, there are insufficient data at the present moment to alter therapy based on HPV status [3]. The most recent 8 th edition of the AJCC Staging Manual has incorporated a separate staging schema for HPV+ oropharyngeal squamous cell carcinoma (OPSCC) as compared to HPV-negative OPSCC, however management recommendations have not been altered despite the separate staging for HPV+ disease [26, 27].

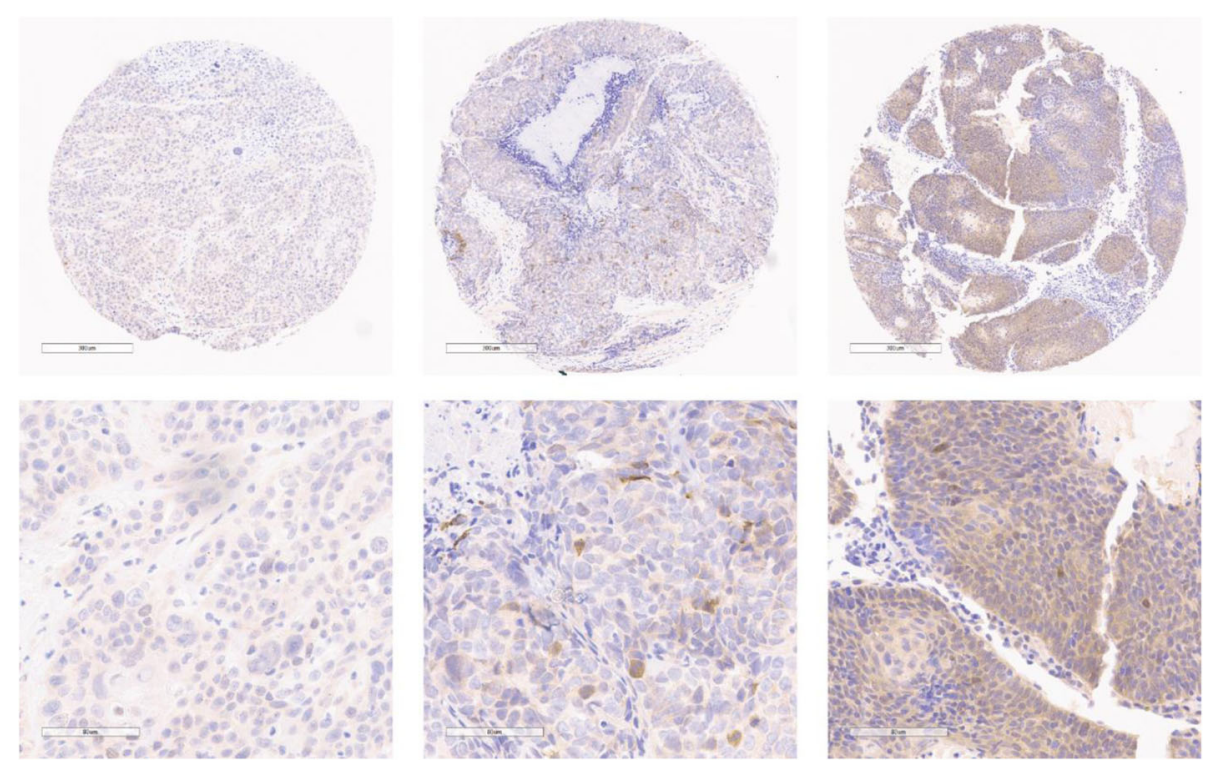

Fig. 3 Representative PAMPK immunostaining of a patient's tumor specimen with low intensity staining (low magnification in top left image and high magnification in bottom left image), medium intensity staining (low magnification in top middle image and high magnification in bottom middle image), and high intensity staining (low magnification in top right image and high magnification in bottom right image) 
Table 4 Correlation between pAMPK activity and positive margin status, ECE, PNI, and LVI

\begin{tabular}{lll}
\hline Outcome & Odds Ratio & $P$-value \\
\hline Positive Margin Status & 1.2 & 0.11 \\
PNI & 1.39 & 0.11 \\
LVI & 1.14 & 0.26 \\
ECE & 1.94 & 0.36 \\
High Risk & 0.9 & 0.79 \\
Low Risk & 1.08 & 0.44 \\
\hline
\end{tabular}

Given the improved prognosis, HPV-associated HNSC $\mathrm{C}$ has been an attractive target to study de-escalation treatment strategies. However, it should be kept in mind that HPV+ patients are a heterogeneous group, with 75$80 \%$ of patients doing very well while the other $20-25 \%$ continuing to have a poor prognosis despite aggressive therapy. Accurate and reliable tools have yet to be identified to help differentiate at the molecular level, and at the time of diagnosis, which patients will do well and which ones will do poorly and more importantly, which subset of patients may benefit from less therapy modalities. The ability to risk stratify patients in this way would be very useful as patients known to do better could then potentially be trialed successfully on deescalation strategies, only subjecting the highest risk patients to intensive multimodality therapy. Randomized trials were recently published comparing cetuximab and RT to cisplatin and RT in HPV positive oropharyngeal cancer and demonstrating inferiority of the cetuximab and RT arm [13, 17]. Perhaps these trials would have benefited from better risk stratification.

We conducted an analysis to try to identify molecular markers within the tumor in HPV-associated head and neck cancer that might help better risk stratify patients and predict recurrence and survival. We analyzed tumor samples from 24 patients and using IHC studied expression of select molecular markers known to be involved in the pathogenesis of HPV+ HNSCC including ERCC1, 4EBP1, AMPK, AKT, S6, PI3K, mTOR, and PTEN. None of these molecular markers portended better outcomes with statistical significance. However, increased pAMPK activation within the tumor was associated with improved RFS with a trend towards statistical significance. Though results were not significant, using the TCGA, there was also a trend that patients with increased AMPK gene expression had longer RFS.

This study was limited by a small sample size. Because our sample size was small, it was difficult to detect meaningful correlations between pAMPK activation and expression of other biomarkers as they relate to recurrence and survival outcomes. It was also difficult to perform adequate subset analyses due to limited sample size, forcing one larger analysis. Further studies into the potential correlation between pAMPK and prognosis are warranted and should be conducted and powered with sample sizes large enough to detect a meaningful difference. Another limitation of this study was that we only looked at a small sample of biomarkers, so there may be others that we didn't evaluate in this study that may have significance in risk stratification. Finally, this study only looked at a cohort of patients that underwent surgery as their primary treatment modality, so conclusions cannot be expanded to patients undergoing radiotherapy as their primary modality of treatment. It would be interesting to do a similar biomarker analysis in the future of these non-surgical patients to compare and contrast with our surgical cohort.

\section{Conclusion}

Increased pAMPK protein and gene expression was associated with improved RFS with a trend toward statistical significance in this study. This data suggests that high pAMPK activation may portend a better prognosis and therefore patients with HPV-associated OPSCC with high-pAMPK expression in their tumors may be more suitable candidates for de-escalation trials. However, these findings require validation in larger studies.

\section{Supplementary information}

Supplementary information accompanies this paper at https://doi.org/10. 1186/s41199-020-00058-2.

Additional file 1: Supplemental Figure 1. Kaplan-Meier plots for RFS in patients with $\mathrm{P} 16+\mathrm{HNSCC}$ with regards to gene expression.

\section{Abbreviations}

BOT: Base of tongue; ECE: Extracapsular extension; HNSCC: Head and neck squamous cell carcinoma; HPV: Human papillomavirus; HR: Hazard ratio; IHC: Immunohistochemistry; LVI: Lymphovascular invasion; OPSCC: Oropharyngeal squamous cell carcinomas; OS: Overall survival; PFS: Progression-free survival; PNI: Perineural invasion; RFS: Relpase-free survival; RT: Radiation therapy; TCGA: The Cancer Genome Atlas; TMA: Tissue microarray; TORS: Transoral robotic surgery

\section{Acknowledgements}

Dr. Wise-Draper was supported by a National Institutes of Health/Translational Science Award KL2 Training Grant TR001426 during a portion of the time this project was conducted. Kelsey Dillehay (PhD), director of the UC Cancer Institute's Tumor Bank and Histopathology Core Laboratory, provided the 26 tumor tissue specimens of patients eligible for this study.

\section{Authors' contributions}

SK helped develop the study's concept and design, applied for IRB approval for the study, identified subjects eligible for the study, obtained clinical information on eligible subjects, contributed to the final analysis for the study, and worked as first author in preparing and editing the final manuscript including figures and tables. SP obtained tissues of eligible subjects from the UC Head and Neck Cancer biorepository, helped secure necessary specific antibodies for $\mathrm{HC}$, and conducted $\mathrm{IHC}$ staining. She provided Fig. 3 and Table 1 for this paper. LR helped put together Table 2 and helped with the final edits of the tables and figures of this paper. CW conducted all analysis of TCGA data and provided the results of this analysis to the final manuscript, including the supplementary figure. JM, ENT surgeon, provided access to the dataset associated with the UC Head and Neck Cancer biorepository from which we found eligible subjects. ZZ and RJ 
conducted all statistical analyses for this study and provided Figs. 1 and 2 . The data that they produced from statistical analysis was used to create Tables 3 and 4. AM assessed intensity and percentage of $\mathrm{IHC}$ staining for each biomarker in this study, and came up with final $\mathrm{IHC} \mathrm{H}$-scores for the study. VT and TWD played major roles in the concept and design of this study. They acted as mentors during each step of this study, and some of TWD's funding was used towards this study. They had major contributions in the final review and editing of the manuscript. The author(s) read and approved the final manuscript.

\section{Funding}

Dr. Wise-Draper was supported by a National Institutes of Health/Translational Science Award KL2 Training Grant TR001426 during a portion of the time this project was conducted, and certain aspects of this study were supported through these funds. Dr. Takiar is supported, in part, by a grant from the United States Department of Veterans Affairs, Biomedical Laboratory Research and Development Service, Career Development Award, IK2 BX004360$01 \mathrm{~A} 1$.

\section{Availability of data and materials}

The datasets created by the IHC analysis of our TMA that were analyzed to create the final results published in this manuscript can be obtained from the corresponding author on reasonable request. The transcriptome data obtained from TCGA can be accessed via the TCGA website. [https://www. cancer.gov/about-nci/organization/ccg/research/structural-genomics/tcga].

\section{Ethics approval and consent to participate}

This is an IRB-approved (University of Cincinnati IRB\#: 2016-0532) retrospective study and included adult patients with HPV-associated OPSCC that underwent definitive surgical resection and are followed at the University of Cincinnati and consented to have their tissue stored in University of Cincinnati's Head and Neck Cancer biorepository. As it was a retrospective study and consent had been previously obtained to store each patient's tissue in our biorepository for research purposes, the need for new consents for this particular study was waived.

\section{Consent for publication}

As data in the manuscript was presented in aggregate, this requirement does not apply to our study.

\section{Competing interests}

The authors declare that they have no competing interests.

\section{Author details}

'Division of Hematology/Oncology, Department of Internal Medicine, Yale University School of Medicine, 333 Cedar Street WWW 201 Attn: Suchin Khanna, New Haven, CT 06510, USA. ²Division of Hematology/Oncology, Department of Internal Medicine, University of Cincinnati College of Medicine, Cincinnati, OH, USA. ${ }^{3}$ Department of Radiation Oncology, University of Cincinnati College of Medicine, Cincinnati, OH, USA.

${ }^{4}$ Department of Otolaryngology, Eastern Virginia Medical Center, Norfolk, VA, USA. ${ }^{5}$ Department of Environmental Health, University of Cincinnati College of Medicine, Cincinnati, OH, USA. ${ }^{6}$ Department of Pathology, UC San Diego Moores Cancer Center, La Jolla, CA, USA.

\section{Received: 29 May 2020 Accepted: 24 August 2020}

Published online: 09 September 2020

\section{References}

1. Global Burden of Disease Cancer Collaboration, Fitzmaurice C, Allen C, Barber RM, et al. Global, regional, and national cancer incidence, mortality, years of life lost, years lived with disability, and disability-adjusted life-years for 32 cancer groups, 1990 to 2015: a systematic analysis for the global burden of disease study. JAMA Oncol. 2017;3(4):524.

2. Chaturvedi AK, Engels EA, Pfeiffer RM, et al. Human papillomavirus and rising oropharyngeal cancer incidence in the United States. J Clin Oncol. 2011;29(32):4294.

3. Vokes EE, Agrawal N, Seiwert TY. HPV-associated head and neck cancer. J Natl Cancer Inst. 2015;107(12):djv344.
4. Jung AC, Briolat J, Millon R, et al. Biological and clinical relevance of transcriptionally active human papillomavirus (HPV) infection in oropharynx squamous cell carcinoma. Int J Cancer. 2010;126:1882-94.

5. Rischin D, Young RJ, Fisher R, et al. Prognostic significance of p16INK4A and human papillomavirus in patients with oropharyngeal cancer treated on TROG 02.02 phase III trial. J Clin Oncol. 2010;28(27):4142.

6. Posner MR, Lorch JH, Goloubeva O, et al. Survival and human papillomavirus in oropharynx cancer in TAX324: a subset analysis from and international phase III trial. Ann Oncol. 2011;22(5):1071.

7. Kumar B, Cipolla MJ, Old MO, et al. Surgical management of oropharyngeal squamous cell carcinoma: survival and functional outcomes. Head Neck. 2016;38(Suppl 1):E1794.

8. Pfister DG, Spencer S, Brizel DM, et al. Head and neck cancers. J Natl Compr Cancer Netw. 2015;13:847-55.

9. Marur S, Li S, Cmelak AJ, et al. E1308: phase II trial of induction chemotherapy followed by reduced-dose radiation and weekly cetuximab in patients with HPV-associated resectable squamous cell carcinoma of the oropharynx - ECOG-ACRIN Cancer Research Group. J Clin Oncol. 2016;35(5): 490-97.

10. Cmelak AJ, Li S, Goldwasser MA, et al. Phase II trial of chemoradiation for organ preservation in resectable stage III or stage IV squamous cell carcinomas of the larynx or oropharynx: results of ECOG 2399. J Clin Oncol. 2007;25(25):3971-7.

11. Chen AM, Felix C, Wang PC, et al. Reduced-dose radiotherapy for HPVassociated SCC of the oropharynx: a single-arm, phase 2 study. Lancet Oncol. 2017;18(6):803-11.

12. Melotek J, Seiwert TY, Blair EA, et al. OPTIMA: a phase II dose and volume de-escalation trial for high- and low-risk HPV+ oropharynx cancers. Ann Oncol. 2019;30(2):297-302.

13. Gillison ML, Trotti AM, Harris J, et al. Radiotherapy plus cetuximab or cisplatin in HPV-positive oropharyngeal cancer: a randomized, multicentre, non-inferiority trial. Lancet. 2019:393(10166):40-50.

14. Rischin D et al. TROG (Trans-Tasman Radiation Oncology Group) 12.01. A randomized trial of weekly cetuximab and radiation versus weekly cisplatin and radiation in good prognosis locoregionally advanced HPV-associated oropharyngeal squamous cell carcinoma. Actual study start date June 2013, estimated primary completion date June 2020, estimated study completion date June 2022.

15. Ferris R et al. ECOG (Eastern Cooperative Oncology Group) 3311 trial. Phase II randomized trial of transoral surgical resection followed by low-dose or standard-dose IMRT in resectable p16+ locally advanceed oropharynx cancer. August 2013 start date, February 2020 estimated primary completion date, February 2023 estimated study completion date.

16. Yom $S$ et al. NRG Oncology Group. NRG-HN002. A randomized phase II trial for patients with p16 positive, non-smoking associated, locoregionally advanced oropharyngeal cancer. Study start date October 2014, estimated primary completion date May 2019, estimated study completion date May 2024

17. Mehanna H, Robinson M, Hartley A, et al. Radiotherapy plus cisplatin or cetuximab in low-risk human papillomavirus-positive oropharyngeal cancer (De-ESCALaTE HPV): and open-label randomized controlled phase 3 trial. Lancet Oncol. 2018;393(10166):51-60.

18. Marur S, D'Souza G, Westra WH, Forastiere AA. HPV-associated head and neck cancer: a virus-related cancer epidemic - a review of epidemiology, biology, virus detection and issues in management. Lancet Oncol. 2010; 11(8):781-9.

19. Leemans $C R$, Braakhuis BJM, Brakenhoff RH. The molecular biology of head and neck cancer. Nat Rev Cancer. 2011;11:9-22.

20. Knuth J, Sharma SJ, Wurdemann N, et al. Hypoxia-inducible factor-1alpha activation in HPV-positive head and neck squamous cell carcinoma cell lines. Oncotarget. 2017:8(52):89681-91.

21. Adams AK, Hallenbeck GE, Casper KA, et al. DEK promoted HPV-positive and negative head and neck cancer cell proliferation. Oncogene. 2015; 34(7):868-77.

22. Bauman JE, Austin MC, Schmidt R, et al. ERCC1 is a prognostic biomarker in locally advanced head and neck cancer: results from a randomized, phase II trial. Br J Cancer. 2013:109(8):2096-105.

23. Kiessling SY, Broglie MA, Soltermann A, et al. Comparison of PI3K pathway in HPV-associated oropharyngeal cancer with and without tobacco exposure. Laryngoscope Investig Otolaryngol. 2018;3(4):283-9.

24. Shackelford DB, Shaw RJ. The LKB1-AMPK pathway: metabolism and growth control in tumor suppression. Nat Rev Cancer. 2009;9:563-75. 
25. McCarty KS, Szabo E, Flowers JL, et al. Use of a monoclonal anti-estrogen receptor antibody in the immunohistochemical evaluation of human tumors. Cancer Res. 1986;46:4244-8.

26. Lydiatt WM, Ridge JA, Patel SG, et al. Oropharynx (p16-) and hypopharynx. In: Amin MB, editor. AJCC cancer staging manual, 8th. New York: Springer; 2017. p. 123

27. O'Sullivan B, Lydiatt WM, Haughey BH, et al. HPV-mediated (p16+) oropharyngeal cancer. In: Amin MB, editor. AJCC cancer staging manual, 8th. New York: Springer; 2017. p. 113.

\section{Publisher's Note}

Springer Nature remains neutral with regard to jurisdictional claims in published maps and institutional affiliations.

Ready to submit your research? Choose BMC and benefit from:

- fast, convenient online submission

- thorough peer review by experienced researchers in your field

- rapid publication on acceptance

- support for research data, including large and complex data types

- gold Open Access which fosters wider collaboration and increased citations

- maximum visibility for your research: over $100 \mathrm{M}$ website views per year

At BMC, research is always in progress.

Learn more biomedcentral.com/submissions 